University of Nebraska - Lincoln

DigitalCommons@University of Nebraska - Lincoln

November 2001

\title{
Exchange Bias in FeF20-CoPt heterosystems with perpendicular anisotropy
}

Christian Binek

University of Nebraska-Lincoln, cbinek@unl.edu

Follow this and additional works at: https://digitalcommons.unl.edu/physicsbinek

Part of the Physics Commons

Binek, Christian, "Exchange Bias in FeF20-CoPt heterosystems with perpendicular anisotropy" (2001). Christian Binek Publications. 42.

https://digitalcommons.unl.edu/physicsbinek/42

This Article is brought to you for free and open access by the Research Papers in Physics and Astronomy at DigitalCommons@University of Nebraska - Lincoln. It has been accepted for inclusion in Christian Binek Publications by an authorized administrator of DigitalCommons@University of Nebraska - Lincoln. 


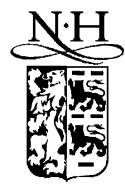

ELSEVIER

\title{
Exchange bias in $\mathrm{FeF}_{2}-\mathrm{Co} / \mathrm{Pt}$ heterosystems with perpendicular anisotropy
}

\author{
Ch. Binek*, B. Kagerer, S. Kainz, W. Kleemann \\ Laboratorium für Angewandte Physik, Gerhard-Mercator-Universität Duisburg, Lotharstr.1 47048 Duisburg, Germany
}

\begin{abstract}
The exchange bias effect is measured in heterosystems with perpendicular anisotropy consisting of $\mathrm{Co} / \mathrm{Pt}$ multilayers on top of the (001) face of a $\mathrm{FeF}_{2}$ single crystal. The resulting exchange field $H_{\mathrm{E}}$ exhibits a strong dependence on temperature and the axial freezing field, $H_{\mathrm{F}}$. Within the framework of an Ising-type model, the $H_{E}$ vs. $T$ as well as the $H_{\mathrm{E}}$ vs. $H_{\mathrm{F}}$ data are explained in terms of the microscopic spin structure at the interface. (C) 2001 Elsevier Science B.V. All rights reserved.
\end{abstract}

Keywords: Exchange biasing; Perpendicular anisotropy; Ising model; Antiferromagnetism

Based on recent progress in the technology of e.g. magnetic field sensors and spin valves [1], there is a renewed interest in the exchange bias effect in layered ferromagnetic (FM)/antiferromagnetic (AF) heterosystems. One of the favored model systems consists of single crystals or textured films of $\mathrm{FeF}_{2}$ which are covered with metallic FM films [2-4]. After field-cooling to below the Néel temperature of $\mathrm{FeF}_{2}, T_{\mathrm{N}}=78 \mathrm{~K}$, the exchange bias effect shifts the hysteresis loop of the FM film by an amount $H_{\mathrm{E}}$ towards the field direction which points opposite to the applied freezing field, $H_{\mathrm{F}}$. In the case of antiferromagnetic exchange coupling between the FM and $\mathrm{AF}$ interface layer, the sign of $H_{\mathrm{E}}$ can change with increasing $H_{\mathrm{F}}$ if the Zeeman energy of the AF interface overcomes the coupling energy. Such competition effects are well known from compensated $\mathrm{FeF}_{2}(110)-\mathrm{Fe}$ bilayers [4]. Recently, we observed a similar decrease of $\left|H_{\mathrm{E}}\right|$ with increasing freezing field in the case of uncompensated $\mathrm{FeF}_{2}(001)$ surfaces covered with $\mathrm{FM} \mathrm{Co} / \mathrm{Pt}$ multilayers [5]. They are deposited at $T=500 \mathrm{~K}$ under UHV conditions by thermal (Co) and electron beam evaporation $(\mathrm{Pt})$ onto the $(001)$ surface of the $\mathrm{FeF}_{2}$ crystal which has been polished to optical flatness with

\footnotetext{
* Corresponding author. Fax: + 49-203-379-1965.

E-mail address: binek@kleemann.uni-duisburg.de (Ch. Binek).
}

$2.5 \mu \mathrm{m}$ diamond paste before transferring the single crystal into the UHV chamber. Such systems exhibit strong uniaxial perpendicular magnetic anisotropy. Hence, freezing fields are applied along the easy axis which points perpendicular to the plane of the $\mathrm{Co} / \mathrm{Pt}$-multilayer. In accordance with the drastic reduction of the degrees of freedom of the spin variables, less complex spin structures are involved in the formation of the FM/AF interface in comparison with the planar anisotropic case. Hence, uniaxial heterosystems are useful candidates for simple Ising-type model considerations, although the observed exchange bias fields of $\left|\mu_{0} H_{\mathrm{E}}\right|<9 \mathrm{mT}$ are surprisingly small.

Fig. 1b shows a hysteresis loop of $\mathrm{FeF}_{2}(001) / \mathrm{Pt} 15 \AA$ $(\mathrm{Co} 3.5 \AA / \mathrm{Pt} 12 \AA)_{5} / \mathrm{Pt} 8 \AA$ measured by SQUID magnetometry after cooling the heterosystem in an axial magnetic field $\mu_{0} H_{\mathrm{F}}=0.1 \mathrm{~T}$ from $T=150$ to $10 \mathrm{~K}$. In order to eliminate all background influences, the exchange bias is safely determined by subtracting the ascending and descending branches of the loops. Fig. 1c shows the result of the subtraction procedure (circles) together with a best Lorentzian fit (solid line). Thus, the exchange bias field $H_{\mathrm{E}}$ is determined with high accuracy from the small shift of the fitting curve with respect to $H_{\mathrm{F}}=0$.

Fig. 1a shows the freezing field dependence of $H_{\mathrm{E}}$ measured at $T=10 \mathrm{~K}$. In addition, Fig. 2 exhibits the temperature dependence of $H_{\mathrm{E}}$. The underlying hysteresis 


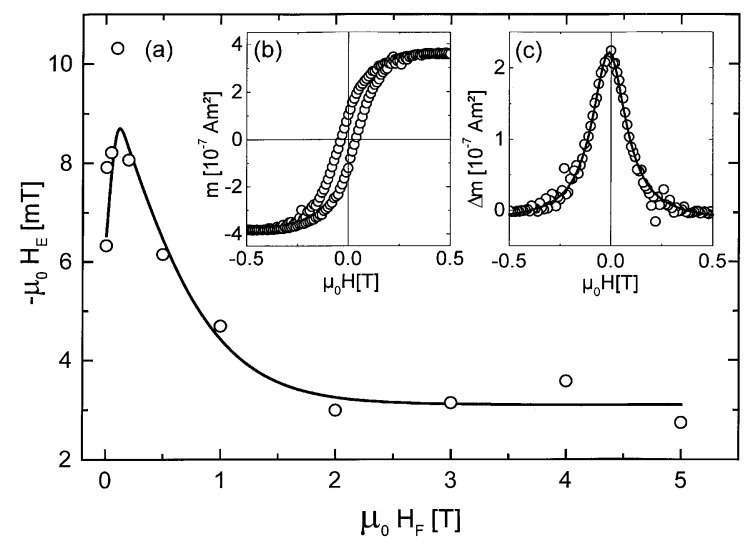

Fig. 1. (a) Experimental data (circles) of the exchange bias $H_{\mathrm{E}}$ obtained from hysteresis loops $m$ vs. $\mu_{0} H$ at $T=10 \mathrm{~K}$ for various freezing fields $H_{\mathrm{F}}$ and the corresponding best fit of Eq. (1) (solid line). Inset (b) shows the hysteresis loop $m$ vs. $\mu_{0} H$ after field cooling at $\mu_{0} H=0.1 \mathrm{~T}$ from $T=150$ to $10 \mathrm{~K}$. Inset (c) shows the subtraction of the ascending from the descending branch of the $m$ vs. $\mu_{0} H$ loop (circles) and the corresponding best Lorentzian fit (solid line).

loops are obtained from measurements at temperatures $2 \mathrm{~K} \leqslant T \leqslant 50 \mathrm{~K}$ after cooling the system in the axial freezing field $\mu_{0} H_{\mathrm{F}}=0.1 \mathrm{~T}$, which maximizes the exchange bias effect (Fig. 1a). Both $H_{\mathrm{E}}$ vs. $H_{\mathrm{F}}$ and $H_{\mathrm{E}}$ vs. $T$ are described by a simple Ising-type model. It takes into account the microscopic spin structure at the interface, which is established on cooling to below $T_{\mathrm{N}}$. Once the spin structure at the interface is stabilized, the underlying antiferromagnet develops its domain structure from the interface into the bulk of the structure. This mechanism makes sure that an uncompensated AF interface with structural roughness is able to generate a net magnetic moment. The frozen magnetic moment $M_{\mathrm{AF}}$ and the field-induced moment $M_{\mathrm{F}}$ of the AF and FM interface layer determine the exchange field according to [6]

$H_{\mathrm{E}} \propto J M_{\mathrm{AF}} M_{\mathrm{F}}$.

In our simple model, we determine $M_{\mathrm{AF}}$ from a thermodynamic analysis, while $M_{\mathrm{F}}$ is completely determined by the applied field. The non-thermal behavior of $M_{\mathrm{F}}$ reflects the high value of the critical temperature $T_{\mathrm{C}} \approx 600 \mathrm{~K}$ of the $\mathrm{Co} / \mathrm{Pt}$ multilayer in comparison with the Néel temperature of $\mathrm{FeF}_{2}$. The thermodynamic analysis of the AF interface is based on the energy function $E=-J S_{\mathrm{F}} S_{\mathrm{AF}}-g \mu_{B} \mu_{0} \mathrm{HS}_{\mathrm{AF}}$, where $J$ is the exchange interaction between spins of the AF interface spin and its fixed FM counterpart. The Zeeman energy of the AF spins competes with the exchange interaction in the case $J<0$. Finally, a second interaction $J^{\prime}$ is introduced. It is assumed [5] to rigidly couple edge spins of the AF interface with ferromagnetic spins, which occupy neigh-

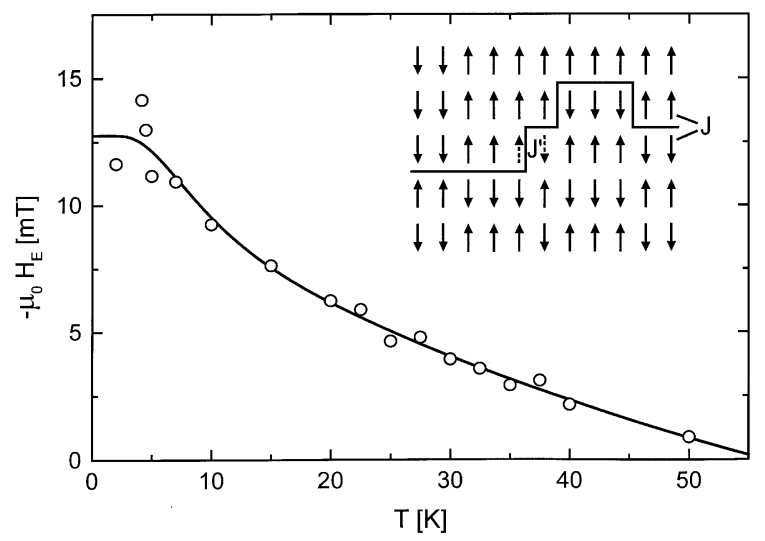

Fig. 2. Experimental data (circles) of the exchange bias $H_{\mathrm{E}}$ for various temperatures $T$ and the corresponding best fit of Eq.(1) extended by the molecular field term $H_{\mathrm{m}}$ (solid line), which enters the magnetic moments $m^{ \pm}$(see text). Inset shows a schematic spin structure of the $\mathrm{FM} / \mathrm{AF}$ interface (line) with two exchange interactions $J$ and $J^{\prime}$ (see text). Arrows indicate the magnetic moments of the FM and AF system, respectively.

boring places of the same atomic layer. Within the framework of these model assumptions the exchange field reads [5]

$$
\begin{aligned}
H_{\mathrm{E}} & \propto\left(2 n_{\mathrm{F}}^{+}-n_{0}\right)\left[n_{\mathrm{F}}^{+}(1-\alpha) \Delta m+(1-\alpha) m^{-}\right. \\
+ & \left.\frac{J^{\prime}}{\left|J^{\prime}\right|} \alpha\left(2 n_{\mathrm{F}}^{+}-n_{0}\right)\right],
\end{aligned}
$$

where $n_{\mathrm{F}}^{+}(H)$ describes the field induced number of upspins in the ferromagnetic interface. $n_{\mathrm{F}}^{+}(H)$ is assumed to be a linear function of $H$ for $|H|<H_{\mathrm{S}}$ which saturates at $n_{0}$ for $H>H_{\mathrm{S}} \cdot m^{ \pm}=g \mu_{\mathrm{B}} \tanh \left(\left( \pm J+g \mu_{\mathrm{B}} \mu_{0} H\right) / k_{\mathrm{B}} T_{\mathrm{N}}\right)$ describes the thermal average of an AF-interface moment which couples to an up $(+)$ or down $(-)$ pointing FM moment. Finally, the roughness parameter $\alpha$ is given by the ratio of the number of moments which are located at steps to the total number of interface moments (see inset Fig. 2). After insertion of Eq. (2) into Eq. (1), the resulting function is fitted to our experimental data (Fig. 1a). The following parameters emerge: $J / k_{\mathrm{B}} T_{\mathrm{N}}=-0.11$, $\mu_{0} H_{\mathrm{S}}=0.08 \mathrm{~T}, \alpha=0.64$ and $g \mu_{\mathrm{B}} / k_{\mathrm{B}} T_{\mathrm{N}}=1.15 \mathrm{~T}^{-1}$. As expected, the microscopic exchange parameter $J / k_{\mathrm{B}} T_{\mathrm{N}}$ is negative. Hence, AF coupling at the interface is favored. Note that the Pt-buffer of $15 \AA$ thickness in $\mathrm{FeF}_{2}(001) / \mathrm{Pt} 15 \AA(\mathrm{Co} 3.5 \AA / \mathrm{Pt} 12 \AA)_{5} / \mathrm{Pt} 8 \AA$ reduces the strength of the interaction by $81 \%$ in comparison

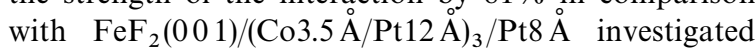
recently [5].

Within a mean-field approach also the temperature dependence of $H_{\mathrm{E}}$ can be modeled. For that purpose, the field dependence of $m^{ \pm}=g \mu_{\mathrm{B}} \tanh \left(\left( \pm J+g \mu_{\mathrm{B}} \mu_{0} H\right) / k_{\mathrm{B}} T\right)$ is extended by a molecular field $H_{\mathrm{m}} \propto\left|T-T_{\mathrm{N}}\right|^{\beta}$, where 
$\beta$ has been fixed during the fitting process as $\beta=0.325$, which is the $3 \mathrm{~d}$ Ising critical exponent of the order parameter. It takes into account the interaction of the AF interface with the subsequent layers of the AF bulk. The molecular field stabilizes the spin structure of the AF interface and, hence, supports the exchange bias field. With increasing temperature $H_{\mathrm{m}}$ decreases which gives rise to a decrease of $H_{\mathrm{E}}$. However, further investigations are necessary in order to obtain quantitative results.

Work supported by Deutsche Forschungsgemeinschaft through Sonderforschungsbereich 491.

\section{References}

[1] B. Dieny et al., Phys. Rev. B 43 (1991) 1297.

[2] J. Nogués, I.K. Schuller, J. Magn. Magn. Mater. 192 (1999) 203.

[3] J. Nogués, T.J. Moran, D. Lederman, I.K. Schuller, K.V. Rao, Phys. Rev. B 59 (1999) 6984.

[4] J. Nogués, D. Lederman, T.J. Moran, I.K. Schuller, Phys. Rev. Lett. 76 (1996) 4624.

[5] B. Kagerer, Ch. Binek, W. Kleemann, J. Magn. Magn. Mater. 217 (2000) 139.

[6] Xi. Haiwen, M.H. Kryder, R.M. White, Appl. Phys. Lett. 74 (1999) 2687. 